\title{
Análisis del consumidor de la tercera edad en el uso de las aplicaciones o APPS de salud
}

\author{
Luis Mañas Viniegra \\ Universidad Complutense y \\ Universidad de Valladolid
}

\begin{abstract}
Palabras clave
Consumidor

tercera edad

app

salud

comunicación

nuevas tecnologías

Internet

Resumen

El consumidor de la tercera edad es cada día más relevante para las marcas, que deben adaptarse a los cambios del macroentorno demográfico. El cuidado de la salud es uno de los ámbitos de interés prioritario para este consumidor y se pretende demostrar que esta tendencia hacia el envejecimiento está consolidada, que su deterioro cognitivo y físico hacen de las apps de salud una herramienta tecnológica que disminuye su dependencia y afianza su seguridad y que el requisito previo para su éxito como producto radica en el equipamiento tecnológico y el uso de Internet. Metodológicamente, se realiza una revisión bibliográfica previa, un análisis de contenido de las apps más populares y se genera información primaria mediante una encuesta que concluye el potencial del producto para el segmento de consumidores elegido a medio plazo.
\end{abstract}

Autor para correspondencia: Luis Mañas Viniegra. Universidad Complutense de Madrid. Facultad de Ciencias de la Información. (Departamento de Comunicación Audiovisual y Publicidad I) Avda Complutense s/n. Correo electrónico: Imanas@ucm.es 


\section{Content Analysis of Elderly Consumer and use of health apps}

\begin{tabular}{c}
\hline Keywords \\
\hline Consumer \\
third age \\
app \\
health communication \\
technology \\
Internet
\end{tabular}

\section{Cómo citar el artículo}

Mañas Viniegra, L. (2015). Análisis del consumidor de la tercera edad en el uso de las aplicaciones o APPS de salud. Revista de Comunicación y Salud, Vol. 5, pp. 69-82.

DOI: http://doi.org/10.35669/revistadecomunicacionysalud.2015.5.69-82 


\section{Introducción}

Las cifras de población a 1 de enero de 2014 publicadas por el Instituto Nacional de Estadística (INE) sitúan en 46.507 .760 habitantes a la población de España, intensificándose el ritmo de descenso de población iniciado en el año 2012. Sin embargo, las mayores tasas de crecimiento se registran en los mayores de 65 años, especialmente los que se encuentran entre los 90 y 94 años $(+7,84 \%)$ y los de entre 70 y 74 años $(5,66 \%)$.

En total, 8.442.887 de personas en España tienen actualmente 65 o más años, representando el $18,15 \%$ del total. Por su parte, los ritmos de crecimiento hacen que el INE haya estimado que en el año 2052, representarán el $37 \%$ de la población en España.

\section{Crecimiento poblacional por grupos de edad}

\begin{tabular}{|c|c|c|c|c|}
\hline \multicolumn{3}{|c|}{ oblación a 1 de ene } & \multirow{2}{*}{$\begin{array}{l}\text { Crecimiento } \\
\text { absoluto }\end{array}$} & \multirow{2}{*}{$\begin{array}{l}\text { Crecimiento } \\
\text { relativo (\%) }\end{array}$} \\
\hline Grupos de eda & & 2014 & & \\
\hline TOTAL & 46.727 .890 & 46.507 .760 & -220.130 & $-0,47$ \\
\hline 0 a 4 años & 2.422 .766 & 2.320 .408 & -102.358 & $-4,22$ \\
\hline 5 a 9 años & 2.440 .531 & 2.478 .051 & 37.520 & 1,54 \\
\hline 10 a 14 años & 2.226 .702 & 2.267 .636 & 40.934 & 1,84 \\
\hline 15 a 19 años & 2.165 .609 & 2.140 .719 & -24.889 & $-1,15$ \\
\hline 20 a 24 años & 2.443 .635 & 2.374 .582 & -69.053 & $-2,83$ \\
\hline 25 a 29 años & 2.899 .633 & 2.747 .345 & -152.288 & $-5,25$ \\
\hline 30 a 34 años & 3.684 .777 & 3.453 .158 & -231.619 & $-6,29$ \\
\hline 35 a 39 años & 4.077.122 & 4.030 .930 & -46.191 & $-1,13$ \\
\hline 40 a 44 años & 3.854 .669 & 3.857 .831 & 3.162 & 0,08 \\
\hline 45 a 49 años & 3.668 .177 & 3.689 .432 & 21.255 & 0,58 \\
\hline 50 a 54 años & 3.284 .958 & 3.333 .708 & 48.750 & 1,48 \\
\hline 55 a 59 años & 2.794 .943 & 2.878 .297 & 83.354 & 2,98 \\
\hline 60 a 64 años & 2.502 .289 & 2.492 .775 & -9.514 & $-0,38$ \\
\hline 65 a 69 años & 2.268 .894 & 2.328 .239 & 59.346 & 2,62 \\
\hline 70 a 74 años & 1.713 .640 & 1.810 .582 & 96.943 & 5,66 \\
\hline 75 a 79 años & 1.726 .105 & 1.652 .850 & -73.255 & $-4,24$ \\
\hline 80 a 84 años & 1.369 .207 & 1.403 .770 & 34.563 & 2,52 \\
\hline 85 a 89 años & 791.817 & 825.438 & 33.621 & 4,25 \\
\hline 90 a 94 años & 308.964 & 333.187 & 24.223 & 7,84 \\
\hline 95 y más años & 83.452 & 88.821 & 5.369 & 6,43 \\
\hline
\end{tabular}

Tabla no 1. Crecimiento Poblacional por grupos de edad. Fuente: INE (2014) 
La Encuesta Continua de hogares elaborada por el INE (2013) indica que en España existen 4.412.000 hogares unipersonales, en los que únicamente vive una persona y, de ellos, en el 40,9\% (1.805.600 personas) residen personas de 65 o más años, siendo mayoritariamente mujeres, en el $72,5 \%$ de los casos. El número de hogares unipersonales creció un 5,2\% de media con respecto al censo de 2011 y un 5,6\% si atendemos exclusivamente a los hogares formados por personas de 65 o más años.

Consecuentemente, cada día se incrementa el número de personas mayores que deberán ocuparse de su salud de forma autónoma, ya que, a pesar de la ayuda que puedan tener de otros familiares o empleados puntualmente, el cuidado de su salud implica el consumo de medicamentos durante varias ocasiones al día, que normalmente produce confusión para identificar cada uno y recordar o no confundir la periodicidad y alternancia de los mismos.

Nos encontramos, por tanto, ante un escenario de fragilidad por parte de esta tipología de consumidor, que Campbell y Wagner (1992) entendieron como "inestabilidad" o incremento en la probabilidad de comenzar un estado de dependencia. No debemos olvidar el impacto que una enfermedad tiene sobre la propia "percepción de las limitaciones físicas, psicológicas, sociales y de disminución de oportunidades" por parte del paciente, como señalan Patrick y Erickson (1993), y que en buena medida "el consumidor está sujeto a muchas influencias que condicionan sus actos", como indica Grande (2002) al señalar la importancia de la influencia de los elementos del entorno y de los internos sobre el consumidor de la tercera edad, que, en general, es "poco propenso a los cambios", en palabras de Pollman y Johnson (1974).

\section{Objetivos e hipótesis}

Este estudio pretende analizar el cambio producido en el macroentorno demográfico como una tendencia consolidada en las últimas décadas, en las que la disminución de los índices de natalidad y el envejecimiento progresivo de la población han provocado que las empresas cada vez más orienten sus productos hacia este segmento de la población en crecimiento. En el caso de las nuevas tecnologías, este escenario abre un abanico de oportunidades para todas las empresas especializadas en el sector salud dirigidas a la tercera edad, gracias al auge del uso de los dispositivos móviles conectados a Internet.

Los objetivos abordados son el análisis del uso de las nuevas tecnologías por parte de la tercera edad, así como el estudio de la utilidad de las apps de salud en este segmento y las variables necesarias para que estas apps consigan ser utilizadas por su público objetivo. El concepto de app de salud queda delimitado en este estudio a aquéllas que guardan relación directa con la medicación y no con la actividad física o deportiva.

Específicamente, se plantean las siguientes hipótesis de partida:

- El envejecimiento de la población se ha convertido en una tendencia consolidada.

- Las nuevas tecnologías mejoran la calidad de vida y el control de la salud de las personas en la tercera edad. 
- El incremento en el equipamiento y uso tecnológico de la tercera edad posibilita que las empresas se especialicen en este segmento de consumidores a medio plazo. El requisito previo es que la tercera edad sea usuaria de dispositivos móviles conectados a Internet.

- La dificultad de uso de las apps de salud son la principal barrera para su adopción por parte del consumidor de la tercera edad.

\section{Metodología}

En cuanto a la orientación metodológica, inicialmente se ha realizado una revisión bibliográfica y de análisis de contenido de fuentes secundarias, principalmente estadísticas, relacionadas con los temas analizados.

En segundo lugar, se ha realizado una investigación cuantitativa basada en una encuesta para generar información primaria, tomando como universo objeto de estudio personas de 65 o más años con un uso de las nuevas tecnologías, al menos, de carácter esporádico. Se ha utilizado el formato de encuesta autoadministrada y autoseleccionada a través de Internet, que ha sido el filtro empleado, ya que implica un uso de las nuevas tecnologías entre las personas de tercera edad. En cuanto a las fechas de recogida, la encuesta estuvo activa en la Red desde el 19 de septiembre hasta el 28 de noviembre de 2014.

El tamaño final de la muestra viene marcado por los 1.064 cuestionarios válidos completados, que representa un margen de error del 3\% para un 95\% de N.C., y que fueron sometidos a un proceso de revisión y validación. La posible existencia de encuestas duplicadas se ha solventado limitando la cumplimentación de una única encuesta por dirección IP. De igual modo, se han eliminado aquéllas que presentan algún tipo de irregularidad, como una presencia mayoritaria de preguntas no contestadas o manifiestamente incongruentes en sus respuestas.

El cuestionario ha sido construido utilizando el plugin FSQM Pro, basado en software libre bajo lenguaje de programación PHP y la muestra ha podido acceder al cuestionario a partir de un enlace facilitado a través de una lista de distribución configurada a partir de las personas de 65 o más años que han facilitado los alumnos de tres universidades públicas: Universidad Complutense de Madrid, Universidad Carlos III de Madrid y Universidad de Valladolid, considerando que el uso tecnológico es mayor en grandes núcleos urbanos. Para comprobar la autenticidad de la identidad de las personas que realizan la encuesta, se han incluido como campos obligatorios el nombre y el número de teléfono, de modo que se han comprobado el $10 \%$ de las identidades y perfiles de los encuestados dentro del control de calidad realizado de la información recabada.

Con el objetivo de ampliar la red de captación de los encuestados, cada vez que alguien rellenaba el cuestionario, tenía la posibilidad de recomendar la realización del mismo a un conocido mediante e-mail, puesto que el filtro consistía en que fuesen usuarios de nuevas tecnologías, al menos, de forma esporádica.

En tercer lugar, se ha realizado un análisis de contenido de las 10 apps de salud más descargadas en las fechas de elaboración del estudio, también denominadas $m$ Health, encontradas para los dos principales sistemas operativos móviles (Android e iOs), se 
han catalogado mediante una ficha de análisis que ha permitido no sólo filtrar las más descargadas, sino eliminar aquéllas que tenían más del $40 \%$ de comentarios negativos y establecer un último filtro por valoración de los usuarios que las habían descargado. Se han seleccionado, de este modo, las siguientes: MedsonTime, RecuerdaMed, MangoHealth, DoseCast, Medisafe, Pillmanager, Medhelper, Mi medicina, Med O'clock, Pillboxie.

\begin{tabular}{|c|c|}
\hline \multirow{5}{*}{$\begin{array}{c}\text { POPULARIDAD } \\
\text { APPS } \\
\text { DE SALUD }\end{array}$} & Número de descargas \\
\hline & $\begin{array}{l}\text { Valoración media } \\
\text { usuarios }\end{array}$ \\
\hline & Número de valoraciones \\
\hline & № comentarios positivos \\
\hline & $\begin{array}{l}\text { № comentarios } \\
\text { neqativos }\end{array}$ \\
\hline
\end{tabular}

Tabla no 2. Ficha técnica de clasificación de apps de salud más populares. Elaboración propia.

Posteriormente, se han realizado algunas capturas de pantalla de la interfaz de uso de esas diez apps para incluirlas en la encuesta realizada y poder, de ese modo, valorar la percepción que existe en el segmento de la tercera edad sobre la facilidad de uso y utilidad de las mismas.

\section{Resultados y discusión}

Internet se ha convertido en el aliado perfecto para la tercera edad en cuanto a consulta de información médica y las apps en cuanto a la planificación de tomas de la medicación y el control de sus hábitos saludables.

Según el informe SESPAS (2012), las personas de 65 a 74 años de edad habían tomado, al menos, un medicamento en las dos semanas previas a la entrevista en el $89 \%$ de los casos y un $93 \%$ en los de 75 o más años, a partir de los datos de la Encuesta Nacional de Salud de 2006.

Además, tendrán que hacerlo durante cada vez más tiempo, ya que la esperanza de vida en España continúa creciendo, especialmente en el caso femenino. Según datos de la Organización Mundial de la Salud, publicados en las Estadísticas Sanitarias Mundiales 2014, las mujeres españolas son las segundas del mundo con mayor esperanza de vida, situada en 85,1 años, y sólo por detrás de Japón, cuyas mujeres alcanzan los 87 años. Por su parte, la esperanza de vida de los hombres españoles se sitúa en 79,3 años.

En sus estimaciones, además, la OMS sitúa a España como el país con mayor envejecimiento en todo el mundo en el año 2050 y situaría a un 40\% de su población con más de 60 años. 
Además del consumo de múltiples fármacos, la tercera edad tiene especial riesgo de padecer Diabetes, siendo este colectivo el que representa el $50 \%$ de todos los casos, según los datos de the Di@bet.es Study (2011).

Por tanto, entre otras muchas necesidades, el paciente mayor de 65 años necesita recibir información de su médico y conocer los medicamentos prescritos. A pesar de ello, SESPAS (2012) indica que casi un $31 \%$ de los pacientes declaran no recibir nunca o casi nunca información de sus médicos, y hasta un $82 \%$ no recibir información escrita. Estos datos no se refieren a la tercera edad, sino a la totalidad de los pacientes, por lo que es probable que en este colectivo sean especialmente altos.

Si los pacientes reciben poca información, entienden menos aún lo que se les dice y necesitan recordar esa información, siendo el olvido otro de los principales males que aquejan a la tercera edad, tenemos los ingredientes que han hecho que Internet se haya convertido en la principal fuente de información sobre salud (sin entrar a valorar la fiabilidad o no según el origen) para la mayoría de los ciudadanos.

Hay que señalar también que el estudio de APEAS (2006) indicó a partir de la revisión de 96.000 consultas de centros de salud en toda España que constataba que existen casi 19 millones de efectos adversos atribuidos a medicamentos, de los cuales más de un millón serían graves y casi la mitad (8,8 millones), potencialmente evitables. Pensemos, en cualquier caso, que los medicamentos pueden tener efectos adversos por los que se tomaron o los que dejaron de tomar.

Sin embargo, en este punto surge una nueva problemática: ¿se encuentra suficientemente extendido el uso de Internet entre la población de 65 o más años? ¿Cuentan con los dispositivos adecuados y saben usarlos? Y si fuera así ¿qué fiabilidad les otorgan y confían en ellos más que en su memoria o son sólo un complemento a la misma?

El principal inconveniente tecnológico, más allá de la mera alfabetización, que continuará solventándose en los próximos años, se encuentra en el poder adquisitivo en los hogares habitados por mayores. CSIC (2014) indica que los hogares con persona de referencia a partir de 65 años "tienen proporcionalmente los menores ingresos de los hogares españoles", aunque "su tasa de riesgo de pobreza se sitúa en 12,2 , inferior a la media de los españoles $(21,6)$ ". En las próximas generaciones de jubilados, este hecho no será tan relevante, puesto que ya contarán con equipos necesarios y mejores conexiones a Internet en el hogar, fruto del envejecimiento de una población hoy sí es mayoritariamente usuaria de internet.

INE (2013) nos muestra en INEBase cómo el grupo de edad inmediatamente anterior a los mayores de 65 años se encuentra habituado en el uso de estas nuevas tecnologías, pero, a pesar de todo, el uso del móvil en la tercera edad actual se encuentra suficientemente desarrollado como para permitir el comienzo de uso de estas apps que ayuden a la reducción de los niveles de dependencia. 


\begin{tabular}{|c|c|c|c|c|}
\hline & TOTAL & $\begin{array}{l}\text { Personas que } \\
\text { han utilizado } \\
\text { alguna vez el } \\
\text { ordenador }\end{array}$ & $\begin{array}{l}\text { Personas que } \\
\text { han utilizado } \\
\text { alguna vez } \\
\text { Internet }\end{array}$ & $\begin{array}{l}\text { Personas } \\
\text { que han } \\
\text { utilizado } \\
\text { alguna vez el } \\
\text { teléfono } \\
\text { móvil en los } \\
\text { últimos } 3 \\
\text { meses }\end{array}$ \\
\hline $\begin{array}{l}\text { DE } 16 \text { a } 24 \\
\text { años }\end{array}$ & $\begin{array}{c}4.139 .16 \\
7\end{array}$ & $99,2 \%$ & $98,5 \%$ & $98,2 \%$ \\
\hline De 25 a 34 años & $\begin{array}{c}6.460 .62 \\
5\end{array}$ & $96,5 \%$ & $96,0 \%$ & $98,8 \%$ \\
\hline De 35 a 44 años & $\begin{array}{c}7.880 .26 \\
9\end{array}$ & $90,5 \%$ & $88,2 \%$ & $98,1 \%$ \\
\hline De 45 a 54 años & $\begin{array}{c}6.937 .88 \\
4\end{array}$ & $79,3 \%$ & $76,0 \%$ & $95,4 \%$ \\
\hline De 55 a 64 años & $\begin{array}{c}5.287 .85 \\
0\end{array}$ & $51,1 \%$ & $51,2 \%$ & $91,0 \%$ \\
\hline De 65 a 74 años & $\begin{array}{c}3.978 .35 \\
5 \\
\end{array}$ & $30,7 \%$ & $25,2 \%$ & $76,8 \%$ \\
\hline TOTAL & $\begin{array}{c}34.484 .1 \\
88 \\
\end{array}$ & $77,5 \%$ & $73,3 \%$ & $94,3 \%$ \\
\hline
\end{tabular}

Tabla n 3. Uso de las tecnologías de información y comunicación por grupos de edad. Fuente: Elaboración a partir de INE, INEBASE (2013)

Las comunidades autónomas que presentan un porcentaje de personas mayores que han utilizado más Internet en los últimos tres meses son: Islas Baleares, Comunidad de Madrid, Cataluña y País Vasco. Extremadura, Galicia y Castilla-La Mancha son las comunidades con menor uso.

En su estudio anual Navegantes en la Red, AIMC (2014) señala cómo el dispositivo más utilizado para acceder a Internet por el usuario general es el móvil, en un $85,5 \%$ de los casos, a pesar de que el dispositivo principal sigue siendo el ordenador por su facilidad para visionar las páginas web. Además, el 70,7\% de los usuarios de Internet mediante móviles reconoce que se conecta varias veces al día con este dispositivo y el $71 \%$ afirma que lo hace repetidamente en menos de una hora. Por tanto, nos encontramos con un escenario favorable para el uso de estas apps de carácter médico. De hecho, el $77,6 \%$ indica que utiliza apps en su móvil o tablet todos o casi todos los días, siendo el $60,2 \%$ el porcentaje de internautas que las utilizan varias veces al día.

Sin embargo, el estudio Navegantes en la Red sólo se refiere al internauta en general, representando las personas de 65 o más años sólo un 2,2\% del total de la muestra, por lo que no disponemos de datos más específicos a partir de la información secundaria analizada.

Si atendemos a los datos expresados en al segunda oleada de 2014 del Estudio General de Medios, el 8,4\% de los usuarios de Internet tienen 65 o más años. El EGM general, por su parte, considera, tomando como universo ese tramo de edad, que un $21,8 \%$ de las personas con 65 o más años son usuarias de internet ayer. Ese 21,8\% 
va a marcar nuestro universo objeto de estudio, en tanto que pretendemos analizar las posibilidades de las apps en un público de la tercera edad ya habituado al uso de Internet.

En cuanto a las actividades realizadas en Internet, dentro del top ten se encuentra "buscar información de salud", citado por el 39\% de los internautas. Este dato, sin duda será considerablemente mayor si atendemos exclusivamente al segmento de la tercera edad.

Y es que, como bien se señala en el estudio de opinión TIC y Dependencia (2007), los enfermos crónicos se enfrentan a una serie de barreras que deben superar para normalizar su vida cotidiana:

- Dependencia de una medicación que les permite mantener una condición sanitaria estable.

- Necesidad de control frecuente o constante de los factores que pueden desencadenar estados críticos.

- Necesidad de mantener una estabilidad en determinados hábitos alimentarios (horario de comidas o cenas, dietas específicas), de medicación (horario de las tomas) o de otra índole que pueden condicionar la vida social del enfermo.

Entre las conclusiones del estudio, se indica que la "telefonía móvil está siendo una herramienta tecnológica muy útil en los últimos años para algunos tipos de enfermos crónicos, que se sienten más seguros disponiendo de este medio. [...] Esta herramienta puede facilitar el contacto con sistemas de información especializados que ayuden al enfermo crónico en su autocontrol". Este estudio abre también un nuevo camino al señalar que "la discapacidad debe plantearse como un hecho que va a afectar temporalmente a la mayoría de las personas". Es decir, que todos en un momento determinado de nuestra vida, la mayoría en nuestra tercera edad, alcanzaremos un grado de dependencia y discapacidad por la reducción progresiva de la autonomía funcional.

Una vez revisada la información secundaria, era necesario aplicarla al consumidor específico de la tercera edad ya usuario regular de Internet, como requisito previo para ser usuario de estas apps de salud, obteniendo algunos resultados en la encuesta realizada que clarifican aún más el estado de la cuestión.

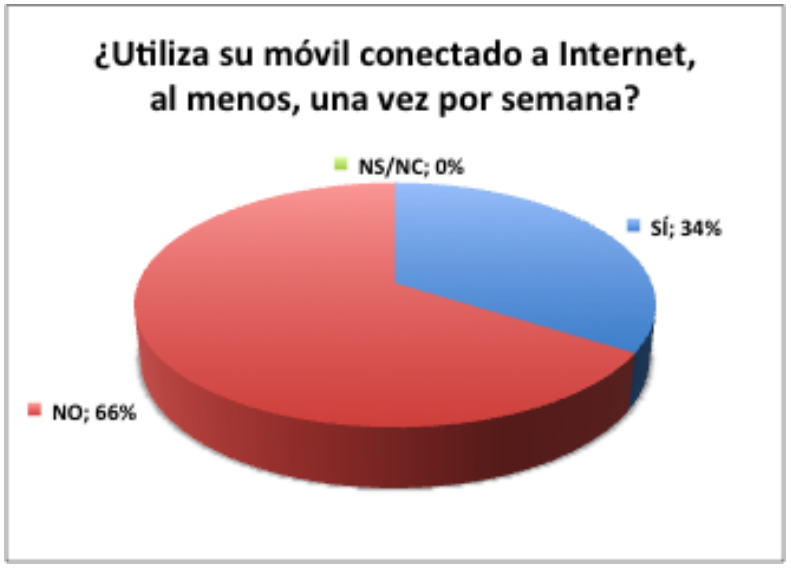

En primer lugar, El $34 \%$ de los mayores de 65 años que han completado la encuesta online (filtro de utilización de Internet) utilizan una tablet o un móvil conectado a Internet con regularidad, es decir, al menos una vez por semana.

Entre las variables por las que eligen estos dos dispositivos frente al ordenador, se encuentran (respuestas 
múltiples) la sencillez de uso (73\% de los usuarios), la comodidad en cuanto al lugar de uso (68\%) o el precio más económico (37\%).

Entre las causas de los no usuarios, destacan (respuestas múltiples) el desinterés $(82 \%)$, la dificultad de uso (34\%) y las limitaciones visuales $(28 \%)$, debido al tamaño de la pantalla.

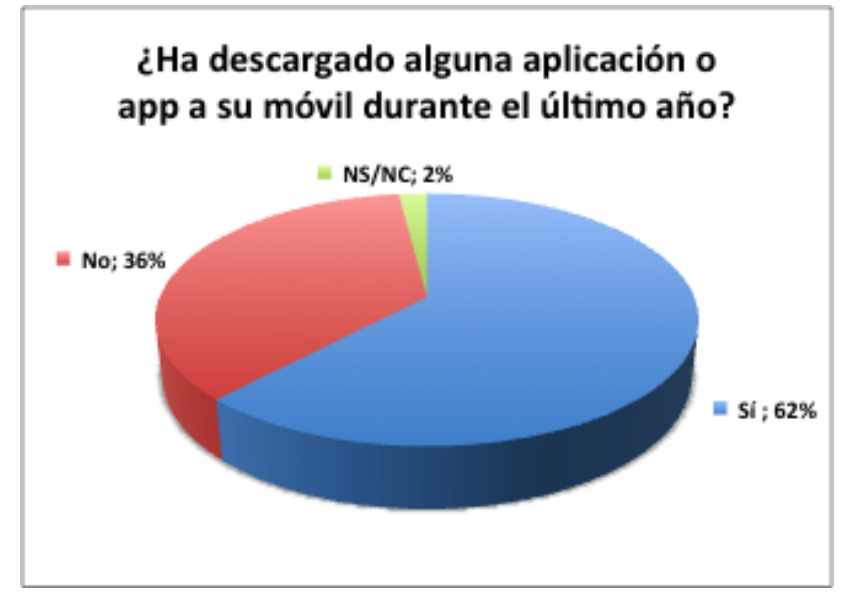

Una vez cumplidos los requisitos de tenencia de dispositivos y de conexión a Internet, destaca un hecho y es que mayoritariamente, en un $62 \%$ de las respuestas, el usuario ha descargado alguna vez una app en su dispositivo móvil, aunque en un $71 \%$ de los casos ha requerido algún tipo de ayuda de familiares 0 amigos.

Sin duda, la experiencia previa en la descarga e instalación de una app de características más sencillas que las de salud, supone un entrenamiento previo que abre la vía a la utilización en una segunda etapa de otras apps como las aquí analizadas. Sus desarrolladores deben trabajar en la simplificación de su uso, la adaptación a las deficiencias visuales y motoras propias de la edad y la promoción de los beneficios que le reportará en su vida diaria.

Efectivamente, la dificultad en su uso parece ser una de las principales barreras en la implantación de las apps de salud en el público de la tercera edad. De hecho, frente al $62 \%$ de encuestados que afirmaban haber descargado alguna app durante el último año, tan sólo el 3\% descargó una app relacionada con la salud.

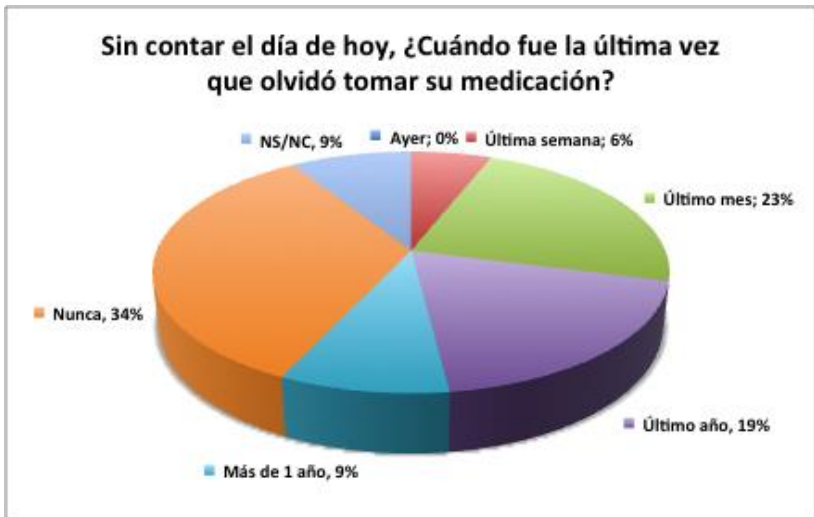

A la hora de analizar la utilidad real que este tipo de apps tienen para el segmento de consumidores escogido, resulta imprescindible conocer si la medicación prescrita es tomada regularmente en las dosis indicadas. Sólo el 34\% se encuentra convencido de que nunca ha olvidado tomar su medicación tal y como la prescribió su médico, por lo que la utilidad real de las apps de salud parecen claras y debemos apuntar a su dificultad de uso como principal barrera una vez más. 
Valore de $\mathbf{1}$ (mínimo) a $\mathbf{5}$ (máximo) la destreza con la que cree que podría utilizar las siguientes aplicaciones o app de salud para su dispositivo móvil

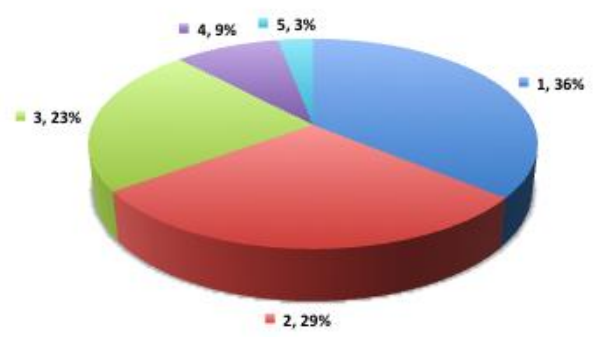

De hecho, al mostrar capturas de pantallas de las diferentes apps de salud catalogadas, sólo el $12 \%$ valoró su destreza con la que percibía que podría utilizarlas para la gestión diaria de su medicación por encima de la valoración intermedia. El $65 \%$ lo hizo por debajo.

¿Supone, por tanto, un problema de uso o un problema de utilidad?

Parece claro que es la dificultad percibida de utilización la barrera establecida hoy en día, puesto que al preguntar sobre si habían consultado alguna vez información sobre salud en Internet, independientemente del dispositivo y de la forma y ocasión de hacerlo, con o sin ayuda, el $72 \%$ afirmaba haber buscado información relacionada con su estado de salud en Internet.

\section{Conclusiones}

Tanto la revisión bibliográfica como la información primaria generada apuntan hacia una serie de conclusiones relacionadas con las perspectivas de crecimiento de las apps de salud en el segmento de consumidores de la tercera edad a medio plazo, que confirman las cuatro hipótesis de partida planteadas:

- La población es España cada día se encuentra más envejecida, registrándose los mayores incrementos de población en los mayores de 65 años y se incrementan cada día más los hogares unipersonales, representando los compuestos por mayores de 65 años el $40,9 \%$.

- En este escenario, es vital que cuenten con una herramienta que les permita cuidar de su salud de manera autónoma, debido al deterioro cognitivo en relación con la toma de medicamentos, habitual en un $89 \%$ de los casos a partir de los 65 años. Este hecho se ve acrecentado porque un $82 \%$ de los pacientes mayores de 65 años afirman no recibir información escrita de su médico, en muchos casos incomprensible por el uso de tecnicismos. Sólo el $34 \%$ se encuentra convencido de que nunca ha olvidado tomar su medicación y las apps de salud son una herramienta adecuada para ello, alcanzando a un segmento de consumidores de interés para las empresas debido a su potencial de crecimiento.

- De los mayores de 65 años que ya son usuarios de Internet, el 34\% utilizan con regularidad un móvil o una tablet conectados a Internet y el $72 \%$ de los encuestados afirman haber buscado información relacionada con su estado de salud en Internet.

- Para que ese crecimiento sea posible, es necesario un uso habitual de Internet, puesto que el principal inconveniente que presentan las apps de salud es la dificultad percibida en su uso por los mayores de 65 años, que actualmente sólo representan el $8,4 \%$ de los usuarios de Internet. El $62 \%$ ha descargado alguna app el último año, aunque haya requerido en la mayoría de los casos ayuda de un conocido. Sin embargo, sólo un 3\% de los que descargaron una app lo hicieron para instalar una de salud, debido principalmente a una barrera 
psicológica: sólo el 12\% percibe que podría utilizarla para la gestión diaria de su medicación con la máxima destreza (valoración de 4 ó 5 sobre 5).

A partir de estas conclusiones, se abren nuevas líneas para posteriores investigaciones, como saber en cuánto tiempo la tercera edad se encontrará preparada para el uso masivo de estas apps de salud, que dependerá en buena medida del envejecimiento de los segmentos de consumidores activos hoy en Internet; dilucidar la mayor importancia que adquirirán unas apps sobre otras, especialmente en lo relacionado con la diabetes por su incidencia en esta población de mayor edad; o aclarar las variables que debe incluir una app de salud en su interfaz para que sea percibida como de fácil utilización por los mayores internautas. Todas estas cuestiones habrá que analizarlas en un estudio comparado, al menos, con los países de nuestro entorno europeo.

\section{Referencias}

Abellán, A., Vilches, J. y Pujol, R. (2014). Un perfil de las personas mayores en España, 2014. Indicadores estadísticos básicos. Informes de envejecimiento en red, № 5. Disponible en http://digital.csic.es/bitstream/10261/101819/1/enredindicadoresbasicos14.pdf. Recuperado el 30 de septiembre de 2014.

AEMPS (2011). Observatorio del Uso de Medicamentos de la AEMPS. Madrid: Agencia Española de Medicamentos y Productos Sanitarios. Disponible en www.aemps.es/profHumana/observatorio/home.htm. Recuperado el 10 de mayo de 2014.

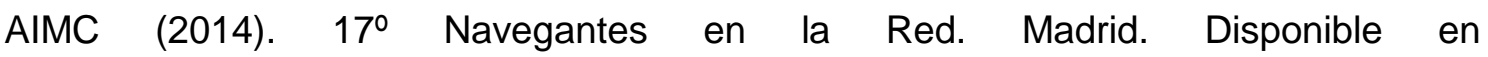
http://download.aimc.es/aimc/J5d8yq/macro2013.pdf. Recuperado el 10 de junio 2014.

AIMC (2014). EGM año móvil octubre 2013 a mayo 2014. Madrid. Disponible en http://www.aimc.es/-Datos-EGM-Resumen-General-.html. Recuperado el 10 de junio de 2014.

AIMC (2014). EGM Audiencias de Internet. $2^{2}$ oleada 2014. Madrid. Disponible en http://www.aimc.es/-Audiencia-de-Internet-en-el-EGM-.html. Recuperado el 10 de junio de 2014.

Alcaide, Juan Carlos (2005). ¿Dónde está el marketing para mayores? Marketing + Ventas, № 205, pp. 46-57.

Andrés-del Campo, Susana y Lima-Maestro, Rosa (2014). Análisis crítico del discurso publicitario institucional/comercial sobre las personas mayores en España. Comunicar, Vol. 21, no 42 , pp. 189-97.

APEAS (2008). Estudio APEAS: Estudio sobre la seguridad de los pacientes en atención primaria de salud. Madrid: Ministerio de Sanidad y Consumo. 
Barca, I.; Parejo, R.; Gutiérrez, P; Fernández, F; Alejandre, G y López, F. (2004). La información al paciente y su participación en la toma de decisiones clínicas. Atención Primaria. Vol. 33, oㅜ 7, pp. 361-7.

Bellón Saameño, J.A. y Martínez Cañabate, T. (2001). La investigación en comunicación y salud. Una perspectiva nacional e internacional desde el análisis bibliométrico. Atención Primaria. Vol. 27, oำ 7, pp. 452-58.

Campbell, A.J. y Buchner, D.M. (1997). Unstable disability and the fluctuations of frailty. Age and ageing. № 26, pp. 315-318

Causapié, Purificación et al (2011). Libro blanco del envejecimiento activo. Madrid: Instituto de Mayores y Servicios Sociales (IMSERSO).

Fernández, J.L., Parapar, C. y Ruiz, M. (2010) El envejecimiento de la población. Lychnos, № 2, pp. 6-11.

Fundación Vodafone (2007). TIC y Dependencia. Estudio de opinión. Madrid: Fundación Vodafone España. Disponible http://tecnologiaedu.us.es/cuestionario/bibliovir/ticee.pdf. Recuperado el 20 de octubre de 2014.

Grande, Ildefonso (2002). El consumo de la tercera edad. Madrid: ESIC.

Grande, Ildefonso (1993). Marketing estratégico para la tercera edad. Madrid: ESIC.

GSMA (2012). Touching lives through mobile health: Assessment of the global market opportunity. Disponible en http://www.gsma.com/connectedliving/wpcontent/uploads/2012/03/gsmapwctouchinglivesthroughmobilehealthreport.pdf.

Recuperado el 18 de agosto de 2014.

Leal Hernández, M., Abellán Alemán, J., Casa Pina, M.T. et al (2004). Paciente polimedicado: ¿conoce la posología de la medicación? ¿Afirma tomarla correctamente? Atención Primaria, Vol. 33, no 8, pp. 451-456.

Madelin, R. (2012). European Directory of Health Apps 2012-2013. Pacient view. Disponible en http://www.patientview.com/uploads/6/5/7/9/6579846/pv_appdirectory_final_web_300812.pdf.

Recuperado el 30 de mayo de 2014.

Patrick, D. y Erickson P. (1993). Health Policy, Quality of Life: Health Care Evaluation and Resource Allocation. New York: Oxford University Press.

Pollman, A.W. y Johnson, A.C. (1974) Resistance to Change. Early Retirement and Managemenal Decisions Industrial Gerontology, 1-1, pp. 33-41.

Sanfélix-Gimeno, Gabriel, Peiró, Salvador y Meneu, Ricard (2012). La prescripción farmacéutica en atención primaria. Informe SESPAS 2014. Gaceta Sanitaria, Vol. 26, supl. 1, pp. 41-45. 
Soriguer F., Goday A. et al (2012). Prevalence of diabetes mellitus and impaired glucose regulation in Spain: the Di@bet.es Study. Diabetologia, № 55, pp. 88-89. Disponible en http://www.ncbi.nlm.nih.gov/pmc/articles/PMC3228950. Recuperado el 18 de septiembre de 2014.

The app intelligence (2014). Informe 50 mejores apps de salud en español. Disponible en http://madrid.theappdate.com/wp-content/uploads/2014/03/Informe-TAD-50Mejores-Apps-de-Salud.pdf. Recuperado el 28 de junio de 2014. 